\title{
Necrotic Wound Caused by Jararaca (Bothrops jararaca) in a Dog - Hyperbaric Oxygen Therapy (HBTO)
}

\author{
Stella Helena Sakata ${ }_{\odot}^{1}$, Marina Frazatti Gallina ${ }^{1}$, Thamires Mizobe ${ }^{2}$, \\ Guilherme Cirino Coelho Pereira ${ }^{1}$, Karina Calixto de Almeida ${ }_{\odot}{ }^{1}$, Vivian Ferreira Zadra ${ }^{3}$, \\ Claudia Tozato Cramer $_{\odot}^{4}$ \& Ivan Felismino Charas dos Santos ${ }^{1}$
}

\begin{abstract}
Background: Snakebites are the main responsible for envenoming in dogs and the bothropic venom remains the most common in Brazil, which can induce a necrotic skin wound. Hyperbaric oxygen therapy (HBOT) use 100\% oxygen under high pressure and used to treat different wounds in human patients. To the authors' knowledge, no reports regarding to use the HBOT in skin wound caused by snakebite (Bothrops jararaca) are present in the literature. The present clinical case aimed to describe the use of HBOT for the treatment of an extensive necrotic wound caused by jararaca snakebite in a dog. Case: A neutered 8-year-old mixed-breed dog, weighing $12 \mathrm{~kg}$, was admitted with a 7-day history of extensive necrotic wound was identified in the face and neck causing by a snakebite, and no sign of pain. The procedure of HBOT (single sessions of $1.5 \mathrm{ATM}, 45 \mathrm{~min}$, repeated every $48 \mathrm{~h}$, up to 12 sessions) was decided, and the complete blood cells, alanine aminotransferase, creatinine, creatine kinase, prothrombin time, activated partial thromboplastin time, wound clinical evaluation were measured at the following time-points: $2^{\text {nd }}, 5^{\text {th }}, 10^{\text {th }}$, and $12^{\text {th }}$ sessions. At the $5^{\text {th }}$ session was identified leukopenia, neutropenia and lymphopenia. Wound re-epithelialization was initiated after the $5^{\text {th }}$ session, and the complete epithelialization was identified at the 12th session of HBOT. During the HBOT no side effects were identified. Three months after the HBOT finished, the animal returned to the clinic and the clinical status evolved positively, and the wound was completed healed.

Discussion: This report described the treatment of an extensive necrotic skin wound caused by snakebite (Bothrops jararaca) in an 8-year-old, neutered, mixed-breed dog using the HBOT. The wound healing was achieved after 12 sessions, similar to the literature, which reported a ranging from 1 to 12 sessions. The HBOT protocol used in this case was similar as reported for human patients with chronic wounds due to the lack of HBOT protocols for animals. No reports regarding the use of HBOT for treat necrotic wound caused by snakebite was described in the literature, and to the authors' knowledge, this is the first report in Brazil describing the use of HBOT in dogs. On the other hand, dogs with surgically induced skin wounds and treated with daily session of HBOT using the treatment protocol of 1.7 ATM (30 min) and 2.0 ATM (40 $\mathrm{min})$ up to $7^{\text {th }}$ day of treatment did not show significant results on healing [9]. This fact was associated with the HBOT achievement in the proliferative and remodeling phases of the healing process. The high intensity of HBOT was between the $5^{\text {th }}$ and $10^{\text {th }}$ session since the wound showed a higher area decrease rate and consequently increase of wound contraction. This period was corresponding to the $10^{\text {th }}$ and $20^{\text {th }}$ day of the healing process, which can be identified angiogenic activity, re-epithelialization, and collagen maturation. The decrease in PVC has been associated with the anticoagulant and/or hemorrhagic activity caused by the venom, and leukopenia, neutropenia and lymphopenia was related with possible bone marrow exhaustion. Single sessions of HBOT (1.5 ATM, 45 min, and repeat each $48 \mathrm{~h}$, up to 12 session) induces healing of necrotic wound caused by snakebite (Bothrops jararaca) in an 8-year-old, neutered, mixed-breed dog without any side effects.
\end{abstract}

Keywords: dog, healing, hyperbaric chamber, skin wound, snake. 


\section{INTRODUCTION}

Bothrops jararaca snakes are the main responsible for envenoming in dogs in Brazil and trigger complexes pathophysiological and inflammatory processes $[6,13,17]$. Bothropic venom has anticoagulant, proteolytic and vasculotoxic action, and the necrotic lesion can be identified in the skin, subcutaneous tissue, muscle, or bones [2,13]. The necrosis is encouraged by a metalloproteinase - jararhagin, which acts by degradation of sub-endothelial matrix proteins leading to the disruption of the blood vessel endothelium $[2,6]$. Systemic symptoms include weakness, anorexia, tachycardia and tachypnea, and hypovolemic shock $[6,17]$. HBOT involves the use of $100 \%$ oxygen under high pressure and is used to treat chronic wounds, radiotherapy wounds, necrotizing fasciitis, and burns, among others [1,7]. In the present clinical case, the use of HBOT for the treatment of an extensive necrotic wound caused by jararaca snakebite in a dog is described. To the authors' knowledge, this is the first report in Brazil describing the use of HBOT for this condition. This report will increment the data regarding the use of HBOT in dogs.

\section{CASE}

An 8-year-old mixed breed neutered dog, weighing $12 \mathrm{~kg}$, living in a rural area, was presented at veterinary clinic, São Paulo, Brazil, with a 7-day history of a wound in the face and neck caused by a jararaca snakebite (Bothrops jararaca) [Figure 1]. The owner reported that the animal had not been treated with any antibiotics or anti-inflammatory, and no physiological changes were observed. On physical examination was identified an extensive necrotic wound in the left side of the face and both sides of the neck (Figure 1) and revealed no pain during palpation. Other clinical parameters remained within the reference.

Complete blood count $(\mathrm{CBC})$ revealed a decrease in PVC (PVC - 35.0\%, normal range: 37.3 - 61.7\%) without any signs of anemia (erythrocytes - $5.5 \mathrm{M} / \mathrm{mL}$, normal range: 5.65 - $8.87 \mathrm{M} / \mathrm{mL}$; hemoglobin - $14.1 \mathrm{~g} / \mathrm{dL}$, normal range: $13.1-20.5 \mathrm{~g} / \mathrm{dL}$ ). The biochemistry tests (alanine aminotransferase, creatinine, creatine kinase), and prothrombin time and activated partial thromboplastin time values remained within the reference limits.

The procedure of HBOT using a hyperbaric chamber for pets $\left(\right.$ Model HVM-M1 $\left.{ }^{\circledR}\right) 1$ [Figure 2] was

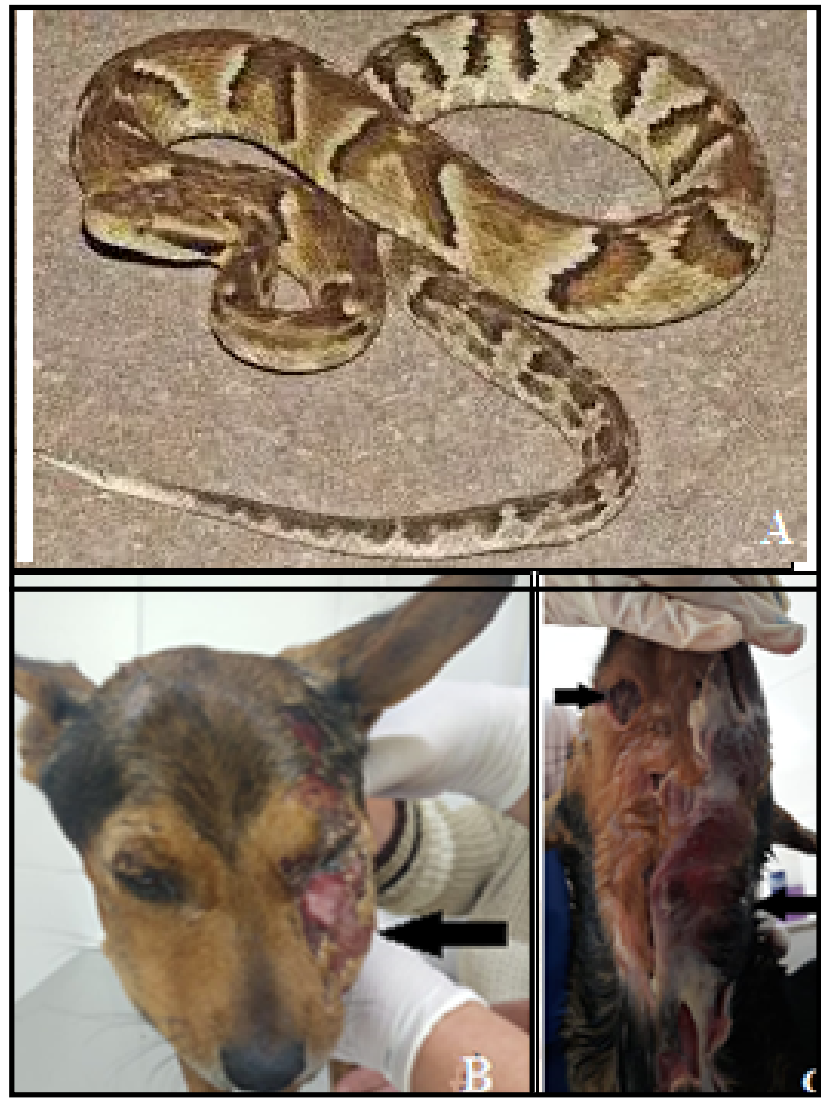

Figure 1. A- Jararaca snake (Bothrops jararaca). B- Extensive necrotic wound in the left side of the face (black arrow) of an 8-year-old, mixedbreed dog, weighing $12 \mathrm{~kg}$. C- Extensive necrotic wound in both sides of the neck (black arrows), 7 days after snakebite.

decided. The treatment protocol consisted of single sessions of 1.5 ATM (45 min) with a compression and decompression pattern of 0.08 ATM per minute and was repeated every $48 \mathrm{~h}$ up to 12 sessions. The treatment was performed in the morning, and between each HBOT session, the wounds were cleaned with chlorhexidine

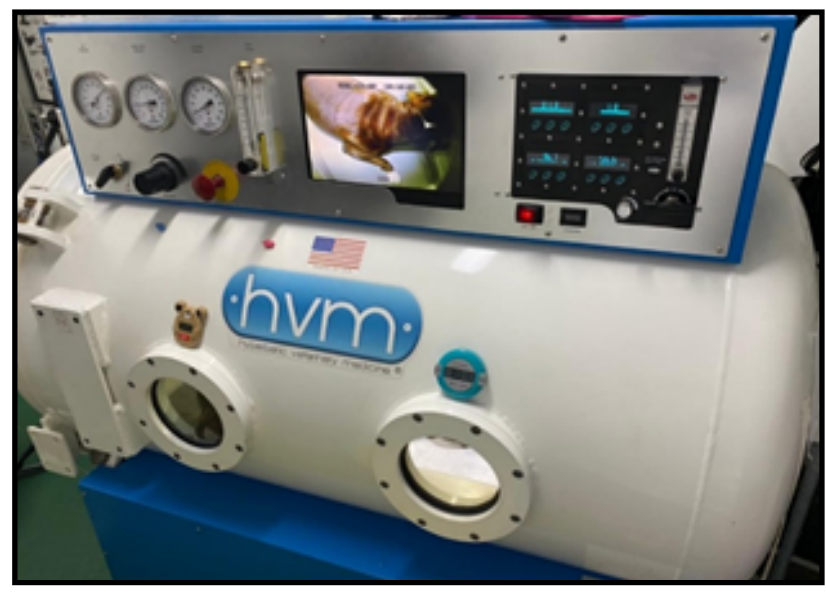

Figure 2. Hyperbaric chamber* used in the treatment of a necrotic wound caused by snakebite (Bothrops jararaca) in an 8-year-old dog. *[Model HVM-M $^{\circledR}$ - length $=1.37 \mathrm{~m}$, external diameter $=83 \mathrm{~cm}$, height $=1.5 \mathrm{~m}$, weight $=300 \mathrm{~kg}$, pressure up to $3 \mathrm{ATM}]$. 
digluconate $0.2 \%\left(\text { Riohex }^{\circledR}\right)^{2}$. No antibiotics or anti-inflammatory were prescribed during the HBOT.

The $\mathrm{CBC}$, alanine aminotransferase, creatinine, creatine kinase, prothrombin time, activated partial thromboplastin time, wound area, and wound contraction values were measured at the following time-points: $2^{\text {nd }}, 5^{\text {th }}, 10^{\text {th }}$, and $12^{\text {th }}$ sessions. The presence of exudate, bleeding, infection and re-epithelialization were determined at the same time-points. The dog was closely monitored for side effects during the compression and decompression phases, $1 \mathrm{~h}$ after each session, and was repeated every $24 \mathrm{~h}$ until finished the HBOT. The wound area was determined using an image software $\left(\text { Image }{ }^{\circledR}\right)^{3}$, and the wound contraction was calculated according to the formula:

Wound contraction $(\%)=\frac{\text { Initial wound area Current wound area }}{\text { Initial wound area }} \times 100 \%$

At the $5^{\text {th }}$ session was identified leukopenia (leucocytes - 3,817/ $\mathrm{mm}^{3}$, normal range: 6,000 $17,000 / \mathrm{mm}^{3}$ ) associated with neutropenia (neutrophils

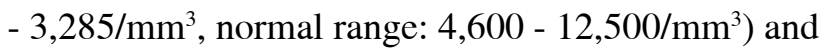
lymphopenia (lymphocytes $-152 / \mathrm{mm}^{3}$, normal range: $800-4,200 / \mathrm{mm}^{3}$ ). Biochemistry and prothrombin time and activated partial thromboplastin time values revealed normal. No changes in the others time-points were identified.

The wound re-epithelialization was initiated after the $5^{\text {th }}$ session, and the complete epithelialization was identified at the $12^{\text {th }}$ session of HBOT (Figure 3 ). During the HBOT no exudate, bleeding, or infection was observed in the wound.

No side effects were identified during the compression and decompression phases. However, the animal presented mild pruritus in the wound 48 $\mathrm{h}$ after the $12^{\text {th }}$ session of HBOT. Topical treatment with a lotion composed of vitamin A, B5, and E was prescribed and was repeated every $12 \mathrm{~h}$. Significant improvement was observed after $24 \mathrm{~h}$. Table 1 showed the wound area and wound contraction values.

The owner reported that the dog eating normally during and after the treatment, no changes were observed regarding behavior. Three months after the HBOT finished, the animal returned to the clinic and the clinical status evolved positively. The wound was healed and was identified presence of hair (Figure 4). $\mathrm{CBC}$, biochemistry, prothrombin time, and activated

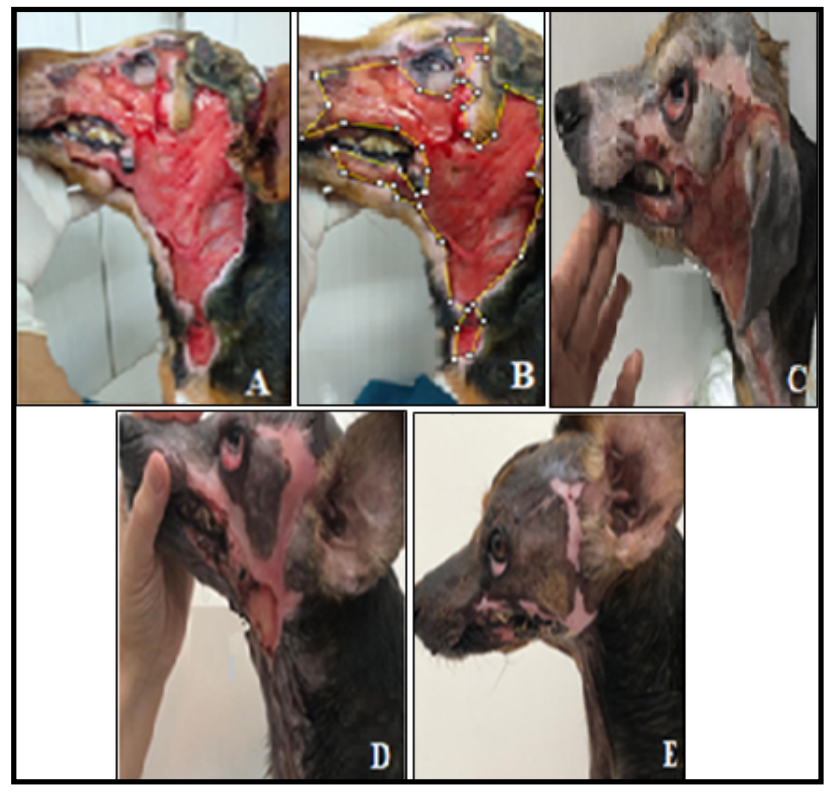

Figure 3. Healing evolution of the extensive necrotic skin wound of an 8 -year-old dog caused by snakebite (Bothrops jararaca) and treated with HBOT. A- Wound aspect in the 2nd session of HBOT showing the absence of necrotic tissue and presence of granulation tissue. B- Measurement of wound area using Image ${ }^{\circledast}$ software. C- Wound aspect in the 5 th session of HBOT. D- Wound aspect in the 10th session of HBO. E- Wound aspect in the 12th session of HBO.

partial thromboplastin time values remained within the reference limits.

\section{DISCUSSION}

This case report described the treatment of an extensive necrotic skin wound caused by snakebite (Bothrops jararaca) in a dog using the HBOT. Wound healing was achieved after 12 sessions, similar to the literature [3], which reported an average of 2.3 sessions, and ranging from 1 to 12 sessions in dogs with different diseases, including the treatment of a necrotic wound with unknown etiology. On the other

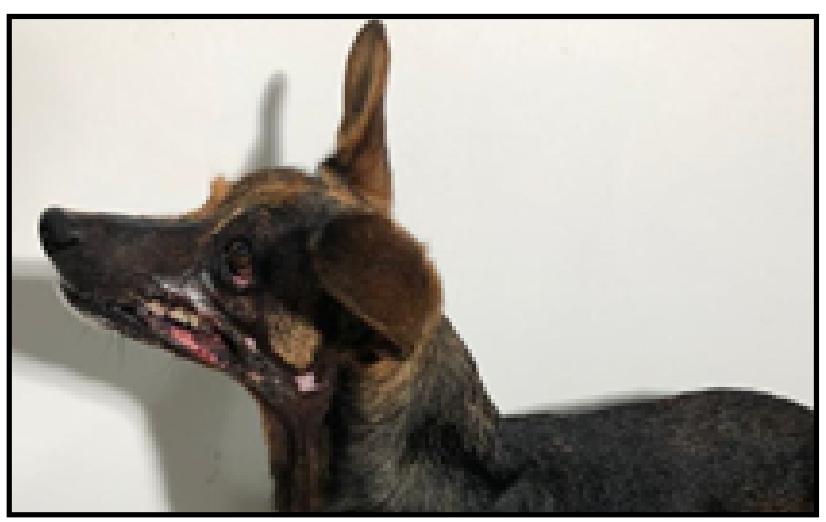

Figure 4. An 8-year-old, mixed-breed dog diagnosed with extensive necrotic skin wound on face and neck caused by snakebite (Bothrops jararaca) after 21 days of HBOT. 
Table 1. Values of the area (mm2) and the wound contraction (\%) of extensive necrotic skin wound caused by snakebite (Bothrops jararaca) and treated by HBOT at following time-points: 2nd session (TP1), 5th session (TP2), 10th sessions (TP3), and in the 12th session (TP4) of HBOT.

\begin{tabular}{ccccc}
\hline & \multicolumn{4}{c}{ Time-points } \\
\hline Parameter & TP1 & TP2 & TP3 & TP4 \\
\hline Wound area $\left(\mathrm{mm}^{2}\right)$ & $8,393.8$ & $6,362.6$ & 820.7 & 0.0 \\
Wound contraction $(\%)$ & 0.0 & 24.2 & 90.2 & 100.0 \\
\hline
\end{tabular}

hand, the hyperbaric chamber used in the present report was for veterinarian use, and no adaptations were needed. The dog of the present report was not treated with antivenom because of the nonexistence scientific evidence that antivenom neutralizes the venom effect in tissue $[2,6,13,17]$.

The novelty of this report concerning in the gap that exists in the literature regarding to the use of HBOT in skin wounds caused by jararaca venom in dogs. Various authors reported the efficacy of HBOT as an adjuvant in the treatment of necrotic wounds associated with snakebites (Bothrops sp.) in human patients $[1,8,15]$. On the other hand, no reports regarding the use of HBOT for treat necrotic wound caused by snakebite was described in the literature, and to the authors' knowledge, this is the first report in Brazil describing the use of HBOT in dogs.

The authors decided to use a similar protocol as reported for human patients with chronic wounds due to the lack of HBOT protocols for animals $[3,8,10,15]$. This fact was related to the high cost of the equipment, and, simultaneously, the lack of reports regarding the use of this therapy $[3,8,15]$. Dogs with surgically induced skin wounds and treated with daily session of HBOT using the treatment protocol of 1.7 ATM (30 $\mathrm{min}$ ) on the first day of treatment following to 2.0 ATM (40 $\mathrm{min}$ ) up to $7^{\text {th }}$ day of treatment did not show significant results on healing [11]. This fact was associated with the HBOT achievement in the proliferative and remodeling phases of the healing process [4].

The high intensity of HBOT was between the $5^{\text {th }}$ and $10^{\text {th }}$ session since the wound showed a higher area decrease rate and consequently increase of wound contraction. This period was corresponding to the $10^{\text {th }}$ and $20^{\text {th }}$ day of the healing process, which can be identified angiogenic activity, re-epithelialization, and collagen maturation [4]. Study reported that HBOT stimulates fibroblasts proliferation and collagen maturation since oxygen is essential for hydroxylation of lysine and proline residues, as well as for protein cross-linking [12]. Others studies showed that, the HBOT promoted vasoconstriction by decreasing vasogenic edema without causing hypoxia; modulated the production of nitric oxide, the vascular endothelial growth factors (VEFG), and inflammatory cytokines $[4,5,14]$.

The presence of pain at the site of snakebite referenced by the literature $[13,17]$ was not identified in our patient. The decrease in PVC has been associated with the anticoagulant and/or hemorrhagic activity caused by the venom $[6,17]$, and the leukopenia, neutropenia and lymphopenia was related with possible bone marrow exhaustion [7,11]. However, these parameters remained in the normal range after the $5^{\text {th }}$ HBOT session. On the other hand, the presence of renal failure, disseminated intravascular coagulation, and shock affected by snakebite (Bothrops sp.) reported in human patients was associated with the amount of venom inoculated by the snake, and the time between the snakebite and treatment $[6,17]$.

The protocol that used in this report was considered safe since no significant side effects were identified, similar to literature [3,4]. These authors considered HBOT safe for dogs and cats when using pressure protocols between 1.5 and $3 \mathrm{ATM}$, and times ranging from 45 to $60 \mathrm{~min}[3,4]$. In human patients, the main serious side effect is related to middle ear barotrauma and toxicity caused by excess oxygen $[4,16]$.

The single sessions of HBOT (1.5 ATM, 45 $\min$, and repeat each $48 \mathrm{~h}$, up to 12 session) induces healing of necrotic wound caused by snakebite (Bothrops jararaca) in an 8-year-old, neutered, mixed-breed dog without any side effects.

\section{MANUFACTURERS}

${ }^{1}$ Hyperbaric Veterinary Medicine. Boca Raton, FL, USA.

${ }^{2}$ Rioquímica Indústria Farmacêutica. São José do Rio Preto, SP, Brazil.

${ }^{3}$ Java Oracle. New York, NY, USA.

Acknowledgments. The authors are grateful to $\mathrm{HVM}^{\circledR}$ and StellaFisiovet Veterinary Clinic, São Paulo, Brazil.

Declaration of interest. The authors report no conflicts of interest. The authors alone are responsible for the contents and writing of the paper. 


\section{REFERENCES}

1 Andrade S.M. \& Santos I.C.R.V. 2016. Hyperbaric oxygen therapy for wound care. Revista Gaúcha de Enfermagem. 37(2): e59257. DOI: https://doi.org/10.1590/1983-1447.2016.02.59257.

2 Baldo C., Jamora C., Yamanouye N., Zorn T.M. \& Moura A.M.S. 2010. Mechanisms of vascular damage by hemorrhagic snake venom metalloproteinases: tissue distribution and in situ hydrolysis. PLoS Neglected Tropical Diseases. 4(6): e727. DOI: https://doi.org/10.1371/journal.pntd.0000727.

3 Birnie G.L., Fry D.R. \& Best M.P. 2018. Safety and tolerability of hyperbaric oxygen therapy in cats and dogs. Journal of the American Animal Hospital Association. 54(4): 188-194. DOI: https://doi.org/10.5326/JAAHA-MS-6548.

4 Braswell C. \& Crowe D.T. 2012. Hyperbaric oxygen therapy. Compendium: Continuing Education for Veterinarians. 34(3): 1-5.

5 Edwards M.L. 2010. Hyperbaric oxygen therapy. part 2: application in disease. Journal of Veterinary Emergency and Critical Care. 20(3): 289-97. DOI: https://doi.org/10.1111/j.1476-4431.2010.00535_1.x.

6 Ferreira Jr.R.S. \& Barravieira B. 2004. Management of venomous snakebites in dogs and cats in Brazil. Journal of Venomous Animals and Toxins including Tropical Diseases. 10(2): 112-132. DOI: https://doi.org/10.1590/S167891992004000200002.

7 Goggins C.A. \& Khachemoune A. 2019. The use of hyperbaric oxygen therapy in the treatment of necrotizing soft tissue infections, compromised grafts and flaps, hidradenitis suppurativa, and pyoderma gangrenosum. Acta Dermatovenerol APA. 28: 81-84. DOI: https://doi.org/10.15570/actaapa.2019.20.

8 Hochedez P., Thomas L. \& Mehdaoui H. 2010. Hyperbaric oxygen therapy after Bothrops lanceolatus snake bites in Martinique: a brief report. Undersea and Hyperbaric Medicine. 37(6): 399-403.

9 Latimer C.R., Lux C.N., Roberts S., Drum M.G., Braswell C. \& Sula M.J.M. 2018. Effects of hyperbaric oxygen therapy on uncomplicated incisional and open wound healing in dogs. Veterinary Surgery. 47(6): 827-836. DOI: https:// doi.org/10.1111/vsu.12931.

10 Mukundan P.K., Ambookan P.V. \& Angappan R. 2016. Hyperbaric oxygen therapy improves outcome of snake envenomation: tertiary center experience. Plastic and Aesthetic Research. 3(2): 59-63. DOI: https://doi.org/10.20517/23479264.2015.11.

11 Mutluoglu M., Cakkalkurt A., Uzun G. \& Aktas S. 2013. Topical oxygen for chronic wounds: a pro/con debate. Journal of the American College of Clinical Wound Specialists. 5(3): 61-65. DOI: https://doi.org/10.1016/j.jccw.2014.12.003.

12 Sander A.L., Henrich D., Muth C.M., Marzi I., Barker J.H. \& Frank J.M. 2009. In vivo effect of hyperbaric oxygen on wound angiogenesis and epithelialization. Wound Repair and Regeneration. 17(2): 179-184. DOI: https://doi. org/10.1111/j.1524-475X.2009.00455.x.

13 Santos M.M.B., Melo M.M., Jacome D.O., Ferreira K.M. \& Habermehl G.G. 2003. Evaluation of local lesions in dogs experimentally envenomed by Bothrops alternatus after different treatments. Arquivo Brasileiro de Medicina Veterinária e Zootecnia. 55(5): 639-644. DOI: http://dx.doi.org/10.1590/S0102-09352003000500020.

14 Schreml S., Szeimies R.M., Prantl L., Karrer S., Landthaler M. \& Babilas P. 2010. Oxygen in acute and chronic wound healing. British Journal of Dermatology. 163(2): 257-268. DOI: http://dx.doi.org/10.1111/j.13652133.2010.09804.x.

15 Schulz R.S., Queiroz P.E.S., Bastos M.C., Miranda E.A., Jesus H.S. \& Gatis S.M.P. 2016. Treatment of wound due to ophidic accident: clinical case. CuidArte Enfermagem. 10(2): 172-179.

16 Shmalberg J., Davies W., Lopez S., Shmalberg D. \& Zilberschtein J. 2015. Rectal temperature changes and oxygen toxicity in dogs treated in a monoplace chamber. Undersea and Hyperbaric Medicine. 42(1): 95-102.

17 Silva L.G., Panziera W., Lessa C.A.S. \& Driemeier D. 2018. Epidemiological and clinical aspects of ophidian bothropic accidents in dogs. Pesquisa Veterinária Brasileira. 38(11): 2150-2154. DOI: https://doi.org/10.1590/16785150-PVB-5889. 